\title{
ON LOCALLY DEFINED FORMATIONS OF SOLUBLE LIE AND LEIBNIZ ALGEBRAS
}

\author{
DONALD W. BARNES
}

(Received 27 October 2011)

\begin{abstract}
It is well known that all saturated formations of finite soluble groups are locally defined and, except for the trivial formation, have many different local definitions. I show that for Lie and Leibniz algebras over a field of characteristic 0 , the formations of all nilpotent algebras and of all soluble algebras are the only locally defined formations and the latter has many local definitions. Over a field of nonzero characteristic, a saturated formation of soluble Lie algebras has at most one local definition, but a locally defined saturated formation of soluble Leibniz algebras other than that of nilpotent algebras has more than one local definition.
\end{abstract}

2010 Mathematics subject classification: primary 17B30; secondary 17A32, 20D10.

Keywords and phrases: Lie algebras, Leibniz algebras, saturated formations, local definition.

\section{Introduction}

In the theory of finite soluble groups, a formation function is a function $f$ which assigns to each prime $p$ a formation $f(p)$ of finite soluble groups. The formation locally defined by $f$ is the class $\operatorname{Loc}(f)$ of all finite soluble groups such that, for each chief factor $H / K$ of $G$ with $p$ dividing $|H / K|$, we have $G / C_{G}(H / K) \in f(p)$. (Here $C_{G}(H / K)$ denotes the centraliser in $G$ of $H / K$.) It is a saturated formation. Apart from the trivial formation, which contains only the group of order one, every saturated formation of finite soluble groups has many such local definitions. This theory is set out in Doerk and Hawkes [4].

If $\Omega$ is a formation of soluble Lie algebras over the field $F$, the formation locally defined by $\Omega$ is the class of Lie algebras $\operatorname{Loc}(\Omega)$ of those Lie algebras such that, for each chief factor $H / K$ of $L$, we have $L / C_{L}(H / K) \in \Omega$. It is a saturated formation. In contrast to the group case, not every saturated formation of soluble Lie algebras is locally defined. The theory of saturated formations of soluble Lie algebras is set out in Barnes and Gastineau-Hills [3]. The analogous theory for Leibniz algebras is set out in Barnes [2].

(c) 2012 Australian Mathematical Publishing Association Inc. 0004-9727/2012 \$16.00 
For finite soluble groups, the formation function $f$ is called integrated if, for all primes $p, f(p) \subseteq \operatorname{Loc}(f)$. It is called full if, for all $p$, every extension of a $p$-group by a group in $f(p)$ is in $f(p)$. For Lie and Leibniz algebras, trivially, $\Re \subseteq \operatorname{Loc}(\Re)$; thus, the analogue of 'integrated' always holds. The significance of the condition 'full' is that if $N$ is a normal $p$-subgroup of a group $G$ and $V$ is an irreducible $G$-module over the field of $p$ elements, then $N$ acts trivially on $V$. There is no condition on an ideal $N$ of a Lie or Leibniz algebra $L$ that ensures that $N$ must act trivially on every irreducible $L$-module. Thus, there is no Lie or Leibniz analogue of the condition 'full'.

\section{Lie algebras over a field of characteristic 0}

The formation $\mathfrak{N}$ of nilpotent Lie algebras is locally defined by the formation $\{0\}$ containing only the zero algebra. If $\Re$ is a nonzero formation, then it contains the formation $\mathfrak{A}$ of abelian algebras. But, if $H / K$ is a chief factor of any soluble Lie algebra $L$ over a field of characteristic 0 , then $L / C_{L}(H / K)$ is abelian. Thus, for any nonzero formation $\Re$, we have $\operatorname{Loc}(\Re)=\subseteq$, the formation of all soluble Lie algebras.

For Lie algebras, in contrast to the case for groups, it is convenient to require that formations be nonempty. If we allow formations to be empty, then the zero formation $\{0\}=\operatorname{Loc}(\emptyset)$ and this is its only local definition.

We thus have, for a field $F$ of characteristic $0, \mathfrak{N}$ and $\subseteq$ are the only locally defined formations, $\mathfrak{N}$ has the unique local definition $\mathfrak{N}=\operatorname{Loc}(\{0\})$ while $\mathfrak{S}=\operatorname{Loc}(\mathfrak{N})$ for any nonzero formation $\mathfrak{R}$. If $F$ is algebraically closed, then $\{0\}, \mathfrak{N}$ and $\subseteq$ are the only saturated formations, but if $F$ is not algebraically closed, then other saturated formations exist. (See Barnes [1].)

\section{Lie algebras over a field of characteristic $p \neq 0$}

Let $F$ be a field of characteristic $p \neq 0$. Then not all saturated formations of soluble Lie algebras over $F$ are locally definable; for example, the formation $\mathfrak{U}$ of supersoluble Lie algebras is saturated but not locally definable. I show that if the saturated formation $\mathfrak{F}$ is locally defined, then the formation $\Omega$ with $\operatorname{Loc}(\Omega)=\mathfrak{F}$ is uniquely determined by $\mathfrak{F}$.

Denote the nil radical of $L$ by $N(L)$. The saturated formation locally defined by $\Re$ consists of those algebras $L$ such that $L / N(L) \in \mathfrak{K}$, algebras which are the extension of a nilpotent algebra $N=N(L)$ by an algebra in $\Omega$. In a widely used terminology, they are nilpotent by $\mathfrak{R}$. It makes sense to denote the locally defined formation by $\mathfrak{N} \Omega$. I reverse this construction.

Definition 3.1. Let $\mathfrak{F}$ be a saturated formation. We define the quotient of $\mathfrak{F}$ by $\mathfrak{M}$ to be

$$
\mathfrak{F} / \mathfrak{N}=\{L / A \mid L \in \mathfrak{F}, A \unlhd L, N(L) \subseteq A\}
$$

Lemma 3.2. Let $\mathfrak{F}$ be a saturated formation of soluble Lie algebras over any field. Then $\mathfrak{F} / \mathfrak{M}$ is a formation. 
Proof. Put $\mathfrak{R}=\mathfrak{F} / \mathfrak{R}$. By its definition, $\Re$ is quotient closed. Suppose that $A_{i} \unlhd L$ and that $L / A_{i} \in \mathfrak{S}, i=1,2$. We have to prove that $L /\left(A_{1} \cap A_{2}\right) \in \mathfrak{F}$. We may suppose without loss of generality that $A_{1} \cap A_{2}=0$.

There exist $X_{i} \in \mathfrak{F}$ with $N\left(X_{i}\right) \subseteq B_{i} \unlhd X_{i}$ and $X_{i} / B_{i} \simeq L / A_{i}$. Then $L / A_{i} \in \mathfrak{F}$, so $L \in \mathfrak{F}$.

We have epimorphisms $\phi_{i}: X_{i} / B_{i} \rightarrow L /\left(A_{1}+A_{2}\right)$ and can identify $L$ with

$$
\left\{\left(y_{1}, y_{2}\right) \in X_{1} / B_{1} \oplus X_{2} / B_{2} \mid \phi_{1}\left(y_{1}\right)=\phi_{2}\left(y_{2}\right)\right\} .
$$

Let $\psi_{i}$ be the composite of the natural homomorphism $X_{i} / N\left(X_{i}\right) \rightarrow X_{i} / B_{i}$ with $\phi_{i}$ and let

$$
X=\left\{\left(x_{1}, x_{2}\right) \in X_{1} / N\left(X_{1}\right) \oplus X_{2} / N\left(X_{2}\right) \mid \psi_{1}\left(x_{1}\right)=\psi_{2}\left(x_{2}\right)\right\} .
$$

Then the natural homomorphism $X_{1} / N\left(X_{1}\right) \oplus X_{2} / N\left(X_{2}\right) \rightarrow X_{1} / B_{1} \oplus X_{2} / B_{2}$ maps the subalgebra $X$ onto $L$. As $\Re$ is quotient closed, it is sufficient to prove that $X \in \Re$. But this is the special case $B_{i}=N\left(X_{i}\right)$.

The chief factors of $X_{i}$ are $\mathfrak{F}$-central irreducible $L$-modules on which $B_{1}$ and $B_{2}$ act trivially. Let $V$ be the direct sum of the chief factors of $X_{1}$ and $X_{2}$. Then the split extension $E$ of $V$ by $L$ is in $\mathfrak{F}$. Since the intersection of the centralisers in $X_{i}$ of the chief factors of $X_{i}$ is $B_{i}$, the intersection of the centralisers in $E$ of the chief factors of $E$ is $V$ and $N(E)=V$. Thus, $L \in \Re$.

The above lemma holds over any field, but the following theorem needs the assumption of nonzero characteristic.

Theorem 3.3. Let $\mathfrak{F}=\operatorname{Loc}(\Re)$ be a locally defined formation of soluble Lie algebras over a field $F$ of nonzero characteristic. Then $\mathfrak{R}=\mathfrak{F} / \mathfrak{N}$.

Proof. Clearly, $\mathfrak{F} / \mathfrak{N} \subseteq \Re$. Suppose $L \in \mathfrak{R}$. By Jacobson [6, Theorem VI.2, p. 205], there exists a faithful, completely reducible $L$-module $V$. Let $V=\oplus V_{i}$, where the $V_{i}$ are irreducible. Let $K_{i}$ be the kernel of the representation of $L$ on $V_{i}$. Since $L / K_{i} \in \Re$, the split extension $E_{i}$ of $V_{i}$ by $L / K_{i}$ is in $\mathfrak{F}$. The representation of $L / K_{i}$ on $V_{i}$ is faithful, so $N\left(E_{i}\right)=V_{i}$, and $L / K_{i} \in \mathfrak{F} / \mathfrak{N}$. But $\bigcap_{i}\left(K_{i}\right)=0$ and it follows that $L \in \mathfrak{F} / \mathfrak{N}$.

\section{Leibniz algebras}

For Leibniz algebras, the situation is a little more complicated than for Lie algebras. A left Leibniz algebra is a linear algebra $L$ in which the left multiplication operators $d_{a}: L \rightarrow L$ given by $d_{a}(x)=a x$ for $a, x \in L$ are derivations. The basic theory of Leibniz algebras is set out in Loday and Pirashvili [5]. The theory of saturated formations of soluble Leibniz algebras is given in Barnes [2].

The subspace $\operatorname{Leib}(L)=\left\langle x^{2} \mid x \in L\right\rangle$ is an abelian ideal of $L$ and $L / \operatorname{Leib}(L)$ is a Lie algebra. Further, $\operatorname{Leib}(L) L=0$. If $V$ is an irreducible $L$-module, then, by a theorem of Loday and Pirashvili [5], $L / C_{L}(V)$ is a Lie algebra and $V$ is either symmetric $(v x=-x v$ for $x \in L$ and $v \in V)$ or antisymmetric $(V L=0)$. It follows that if $\mathfrak{F}=\operatorname{Loc}(\Omega)$ is a locally defined formation of soluble Leibniz algebras, then $\mathfrak{F}=\operatorname{Loc}\left({ }^{\operatorname{Lie}} \mathfrak{R}\right)$, where ${ }^{\operatorname{Lie}} \Re$ is the class of all Lie algebras in $\Re$. 
The characteristic 0 case is very like that of Lie algebras. The saturated formation $\mathfrak{N}$ of nilpotent Leibniz algebras is locally defined by the zero formation. If $\mathfrak{R}$ is a nonzero formation, then it contains the abelian algebras and $\operatorname{Loc}(\Omega)=\subseteq$, the class of all soluble Leibniz algebras. As for Lie algebras, $\mathfrak{N}$ and $\mathfrak{\subseteq}$ are the only locally defined formations.

The case of characteristic $p \neq 0$ is closely tied to the corresponding case for Lie algebras. By Barnes [2, Corollary 3.17], if $\mathfrak{F}$ is a saturated formation of soluble Leibniz algebras, then ${ }^{\mathrm{Lie}} \mathfrak{F}$ is a saturated formation of soluble Lie algebras and we have a one to one correspondence $\mathfrak{F} \leftrightarrow{ }^{\operatorname{Lie}} \mathfrak{F}$. If $\mathfrak{F}=\operatorname{Loc}(\Re)$, then both $\mathfrak{F}$ and ${ }^{\operatorname{Lie}} \mathfrak{F}$ are locally defined in the corresponding categories by ${ }^{\mathrm{Lie}} \mathfrak{R}$. We thus have the following theorem.

THEOREM 4.1. Let $\mathfrak{F}$ be a locally defined formation of soluble Leibniz algebras over a field of characteristic $p \neq 0$. Then there exists a unique formation $\mathrm{S}_{0}$ of soluble Lie algebras with $\mathfrak{F}=\operatorname{Loc}\left(\Omega_{0}\right)$.

However, except for the case $\mathfrak{N}=\operatorname{Loc}(\{0\})$, there are always other formations $\mathfrak{R}$ of soluble Leibniz algebras with $\operatorname{Loc}(\Re)=\operatorname{Loc}\left(\Re_{0}\right)$.

Lemma 4.2. Let $\Omega_{0} \neq\{0\}$ be a formation of soluble Lie algebras. Let $P_{1}, \ldots, P_{n}$ be soluble Leibniz algebras such that $P_{i} / \operatorname{Leib}\left(P_{i}\right) \in \Omega_{0}$ and let $\Omega$ be the smallest formation of soluble Leibniz algebras containing $\mathfrak{R}_{0}$ and the algebras $P_{i}$. Then Lie $\Omega=\Omega_{0}$ and $\operatorname{Loc}(\Re)=\operatorname{Loc}\left(\Omega_{0}\right)$.

Proof. We have to show that, if $L \in \Re$, then $L / \operatorname{Leib}(L) \in \Omega_{0}$. Since $L \in \Re$, there exist ideals $N_{1}, \ldots, N_{r}$ of $L$ with $\bigcap_{i} N_{i}=0$ such that, for each $i$, either $L / N_{i} \in \mathfrak{R}_{0}$ or $L / N_{i} \simeq P_{j}$ for some $j$. We have the natural epimorphisms $\phi_{i}: L \rightarrow L / N_{i}$ and $L$ may be regarded as the subalgebra of $\bigoplus_{i} L / N_{i}$ whose elements are the $\left(\phi_{1}(x), \ldots, \phi_{r}(x)\right)$ for $x \in L$. But $\phi_{i}$ maps $x^{2}$ to $\phi_{i}(x)^{2}$, thus maps $\operatorname{Leib}(L)$ onto $\operatorname{Leib}\left(L / N_{i}\right)$ and so gives an epimorphism $\psi_{i}: L / \operatorname{Leib}(L) \rightarrow\left(L / N_{i}\right) / \operatorname{Leib}\left(L / N_{i}\right)$. This expresses $L / \operatorname{Leib}(L)$ as a subdirect sum of the $\left(L / N_{i}\right) / \operatorname{Leib}\left(L / N_{i}\right)$. But, for all $i,\left(L / N_{i}\right) / \operatorname{Leib}\left(L / N_{i}\right) \in \Omega_{0}$. As $\psi_{i}(L / \operatorname{Leib}(L)) \in \Omega_{0}$ for all $i, L / \operatorname{Leib}(L) \in \mathfrak{S}_{0}$, as asserted.

We can always construct Leibniz algebras $P$ with $P / \operatorname{Leib}(P) \in \Omega_{0}$. Let $L$ be any Lie algebra in $\Omega_{0}$ and let $V$ be any nontrivial left $L$-module. We make this into a Leibniz module by defining $V L=0$. Let $P$ be the split extension of $V$ by $L$. Then $P$ is not a Lie algebra, so not in $\Omega_{0}$. We have $\operatorname{Leib}(P)=V$ and so $P / \operatorname{Leib}(P) \simeq L \in \mathfrak{S}_{0}$. Thus, we can always produce $\Re \neq \Omega_{0}$ with $\operatorname{Loc}(\Re)=\operatorname{Loc}\left(\Omega_{0}\right)$.

\section{References}

[1] D. W. Barnes, 'Saturated formations of soluble Lie algebras in characteristic 0', Arch. Math. (Basel) 30 (1978), 477-480.

[2] D. W. Barnes, 'Schunck classes of soluble Leibniz algebras', arXiv:1101.3046 (2011).

[3] D. W. Barnes and H. M. Gastineau-Hills, 'On the theory of soluble Lie algebras', Math. Z. 106 (1968), 343-354.

[4] K. Doerk and T. Hawkes, Finite Soluble Groups (De Gruyter, Berlin-New York, 1992). 
[5] J.-L. Loday and T. Pirashvili, 'Leibniz representations of Lie algebras', J. Algebra 181 (1996), 414-425.

[6] N. Jacobson, Lie Algebras (Interscience, New York, 1962).

DONALD W. BARNES, 1 Little Wonga Road, Cremorne, NSW 2090, Australia e-mail: donwb@iprimus.com.au 